\title{
Coherency: An Innovation to Test Data Quality and Reduce Comparisons in the ANP
}

\author{
Cooper, Orrin ${ }^{\mathrm{a}} \&$ Yavuz, Idil ${ }^{\mathrm{b}}$ \\ ${ }^{a}$ University of Memphis, 332 Fogelman College Business Admin Bldg. Memphis, TN 38152, \\ ${ }^{b}$ Department of Statistics, School of Science, Dokuz Eylul University, Tinaztepe, Buca, Izmir, Turkey \\ olcooper@memphis.edu
}

\begin{abstract}
Saaty recognized the value of research that would improve the quality of decision data. The Linking Coherency Index (LCI) is an innovative method to test for coherency in ANP Supermatrices. Coherent data can be defined as self-consistent and noncontradictory with respect to a particular system. Coherency can also be thought of as a "super consistency test" or a test for consistency at the level of the entire Supermatrix. Linking Estimates (LE) are an important component used to calculate the LCI and can also be used to reduce the number of comparisons that are required in ANP decisions. The value of testing the coherency of the Supermatrix and reducing comparisons will be demonstrated through a neat example.
\end{abstract}

Keywords: Coherency, Consistency, Linking Coherency Index (LCI), Linking Estimate

\section{Introduction}

From the beginning, Saaty recognized that tests that would improve the quality of decisions were worth pursuing, "As yet there is no statistical theory (to the best of our knowledge) which would assist us in deciding how well judgmental data correspond to reality. ... It is clear that this is an area of research that is worth pursuing" (1977, p. 247 emphasis added). Coherency is one example of an innovative and important data quality check that tests a sort of consistency at the level of the entire Supermatrix (Cooper \& Yavuz, 2016; Yavuz \& Cooper, 2017). Coherent data can be defined as self-consistent and non-contradictory with respect to a particular system. Coherency is a crucial test that should be performed when using the Analytic Network Process (ANP); and by testing for coherency decision makers can have increased confidence in their decisions. Taking advantage of the relationships used to test for coherency also has the potential to provide a way to reduce the number of comparisons required in an ANP decision model.

\section{Literature Review}

Consistency is an important test that can be used to test and improve the quality of an AHP/ANP decision (Brunelli, Canal, \& Fedrizzi, 2013). Consistency has been a part of the AHP from the beginning. Many different types of consistency have been identifed and methods have been developed to test at the level of the pairwise comparision matrix 
(PCM) for the different types of consistency. What if consistency could be checked not only at the level of the PCM but also at the level of the Supermatrix? Schoner, Wedley, \& Choo (1993) developed the linking pin approach which is a crucial component in the calculation of the Linking Estimates (LE) that are used to calculate the Linking Coherency Index (LCI). Decision makers cannot ignore the fact that each priority vector has a unit of measurement. Any conversions made among the prioirity vectors must be made with respect to the specific units in each priority vector. The linking pin approach relies on the structural dependence of the decision to make conversions not only possible but also meaningful. The LCI was developed to test a level of super consistency or consistency at the level of the Supermatrix (Cooper \& Yavuz, 2016; Yavuz \& Cooper, 2017). Coherency has also been suggested as a potential solution to the sheer number of comparisons that can be required for an ANP decision. Incomplete priority vectors are by definition incoherent and therefore could be inferred by sampling from the LE. Exploiting this conclusion one can then reduce the number of comparisons needed to make an decision using the ANP.

\section{Research Design/Methodology}

The ability to reduce the number of comparisons needed in an ANP model will be demonstrated through an example after showing how the LE and LCI are calculated. The current approach to obtain the LE and LCI is as follows. Assume the Supermatrix $\hat{S}$ represents a sample decision where three alternatives are evaluated with respect to six criteria which are organized into two clusters with three criteria in each cluster:

$\hat{S}=\begin{gathered}C_{1.1} \\ C_{1.2} \\ \vdots \\ C_{2.3} \\ A_{1} \\ A_{2} \\ A_{3}\end{gathered}\left[\begin{array}{ccccccc}C_{1.1} & C_{1.2} & \ldots & C_{2.3} & A_{1} & A_{2} & A_{3} \\ 0 & 0 & \ldots & 0 & x_{A_{1}, 1.1} & x_{A_{2}, 1.1} & x_{A_{3}, 1.1} \\ 0 & 0 & \ldots & 0 & x_{A_{1}, 1.2} & x_{A_{2}, 1.2} & x_{A_{3}, 1.2} \\ \vdots & \vdots & \ddots & \vdots & \vdots & \vdots & \vdots \\ x_{C_{1.1}, 1} & x_{C_{1.2}, 1} & \ldots & x_{C_{2.3}, 1} & 0 & 0 & 0 \\ x_{C_{1.1}, 2} & x_{C_{1.2}, 2} & \ldots & x_{C_{2.3}, 2} & 0 & 0 & 0 \\ x_{C_{1.1}, 3} & x_{C_{1.2}, 3} & \ldots & x_{C_{2.3}, 3} & 0 & 0 & 0\end{array}\right]$.

Every column of the Supermatrix should be converted to a new ratio by dividing the components of each vector by any common element within the priority vectors; the first element in each priority vector will be used herein. The resulting matrix will now have the form: 


\begin{tabular}{|c|c|c|c|c|c|c|c|}
\hline & $C_{1.1}$ & $C_{1.2}$ & $\ldots$ & $C_{2.3}$ & $A_{1}$ & $A_{2}$ & $A_{3}$ \\
\hline$C_{1.1}$ & 0 & 0 & $\cdots$ & 0 & $\frac{x_{A_{1}, 1,1}}{x_{A_{1}, 1,1}}=1$ & 1 & 1 \\
\hline$C_{1.2}$ & 0 & 0 & $\ldots$ & 0 & $\frac{x_{A_{1}, 1,2}}{x_{A_{1}, 1,1}}$ & $\frac{x_{A_{2}, 1,2}}{x_{A_{2}, 1.1}}$ & $\frac{x_{A_{3}, 1.2}}{x_{A_{3}, 1.1}}$ \\
\hline$\vdots$ & $\vdots$ & $\vdots$ & $\ddots$ & $\vdots$ & $\vdots$ & $\vdots$ & $\vdots$ \\
\hline$C_{2.3}$ & 0 & 0 & & 0 & $\frac{x_{A_{1}, 2.3}}{x_{A_{1}, 1.1}}$ & $\frac{x_{A_{2}, 2,3}}{x_{A_{2}, 1.1}}$ & $\frac{x_{A_{3}, 2.3}}{x_{A_{3}, 1.1}}$ \\
\hline$A_{1}$ & $\frac{x_{C_{1,1,1}}}{x_{C_{1.1}, 1}}=1$ & 1 & $\ldots$ & 1 & 0 & 0 & 0 \\
\hline$A_{2}$ & $\frac{x_{C_{1,1,2}}}{x_{C_{1,1}, 1}}$ & $\frac{x_{C_{1,2,2}}}{x_{C_{1,2}, 1}}$ & $\cdots$ & $\frac{x_{C_{2,3,2}}}{x_{C_{2,3}, 1}}$ & 0 & 0 & 0 \\
\hline$A_{3}$ & $\frac{x_{C_{1,1,3}}}{x_{C_{1,1}, 1}}$ & $\frac{x_{C_{1,2,3}}}{x_{C_{1,2}, 1}}$ & $\cdots$ & $\frac{x_{C_{2,3,3}, 3}}{x_{C_{2,3}, 1}}$ & 0 & 0 & 0 \\
\hline
\end{tabular}

If the entries from one column in the lower left-hand side of the Supermatrix are used as the links to convert the entries in the upper right-hand side of the Supermatrix into quantities of a single unit, the converted upper right-hand side will be in this form:

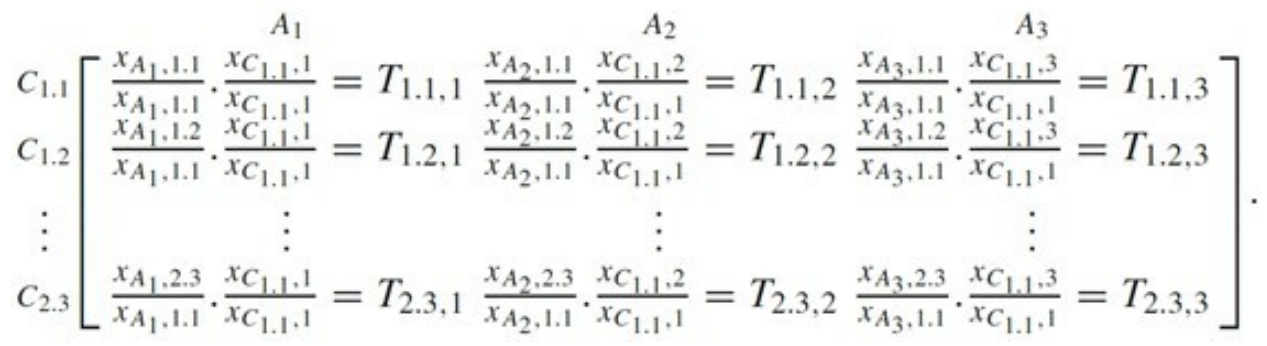

With each entry in each column now represented in the units of a particular, yet same, ratio they can be aggregated and combined to obtain a new estimate of $S$ which we call a Linking Estimate (LE). This new Supermatrix will be notated by $S_{C 1.1}^{L}$ since the criterion $C_{1.1}$ was used as the link. This estimate can be obtained by performing the following calculations: 


$$
\begin{aligned}
& \begin{array}{lllllll}
C_{1.1} & C_{1.2} & \ldots & C_{2.3} & A_{1} & A_{2} & A_{3}
\end{array}
\end{aligned}
$$

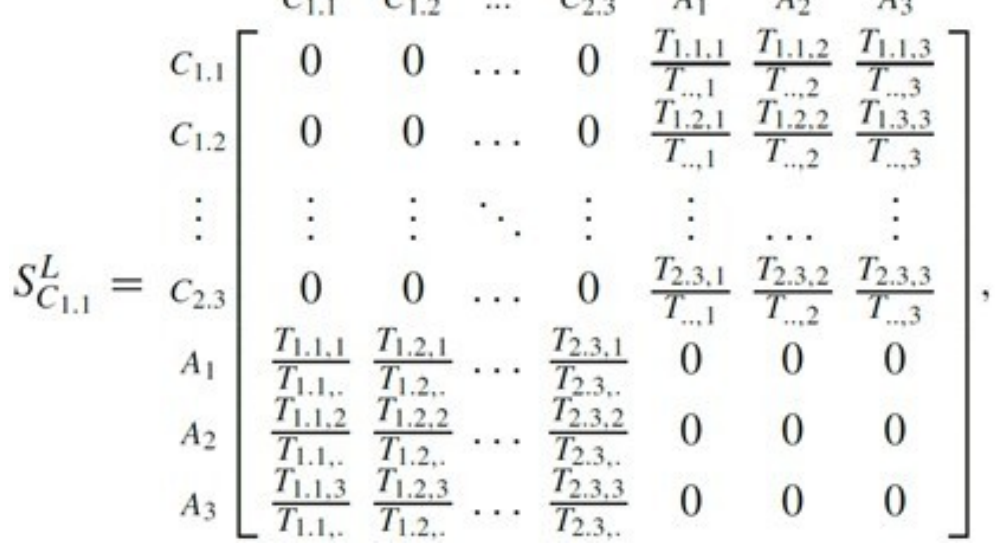

where $\quad T_{i, j, .}=\sum_{n=1}^{3} T_{i, j, n} \quad$ and $\quad T_{., n}=\sum_{i=1}^{2} \sum_{j=1}^{3} T_{i, j, n} . \quad$ The same process can be repeated $n+m$ times where $n$ is the number of alternatives and $m$ is the number of criteria; each time a different criterion or alternative is chosen as the link, resulting in $n+m$ linking estimates (LE).

The LE can then be used to calculate the LCI as follows:

$$
L C I_{b c}= \begin{cases}\frac{1}{\left(n_{\text {alts }} * n_{\text {crit }}\right)} \sum_{i=n_{\text {crit }}+1}^{n_{\text {alts }}+n_{\text {crit }}} \sum_{j=1}^{n_{\text {crit }}} \frac{\max \left(S_{b,(i j)}^{L}, S_{c,(i j)}^{L}\right)}{\min \left(S_{b,(i j)}^{L}, S_{c,(i j)}^{L}\right)}, & \text { if } b, c \in \Omega_{1}, \\ \frac{1}{\left(n_{\text {alts }} * n_{\text {crit }}\right)} \sum_{i=1}^{n_{\text {crit }}} \sum_{j=n_{\text {crit }}+1}^{n_{\text {crit }}+n_{\text {alts }} \frac{\max \left(S_{b,(i j)}^{L}, S_{c,(i j)}^{L}\right)}{\min \left(S_{b,(i j)}^{L}, S_{c,(i j)}^{L}\right)},} & \text { if } b, c \in \Omega_{2}, \\ \text { undefined } & \text { if } b \in \Omega_{1}, c \in \Omega_{2} \\ & \text { or } b \in \Omega_{2}, c \in \Omega_{1}\end{cases}
$$

where $\Omega_{1}$ and $\Omega_{2}$ are the sets containing the alternatives and criteria respectively and $S_{i,(i j)}^{L} \quad$ represent the entry in the $i$ th row and $j$ th column of the corresponding LE matrix.

\section{Data/Model Analysis}

This weighted Supermatrix represents a decision model with four alternatives evaluated with respect to six criteria that are equally distributed among two criteria clusters. This Supermatrix contains priority vectors which are incoherent (red) and for which the decision maker intends to use the LCI and LE to infer (yellow) in order to reduce the number of comparisons that would be required to finish filling in the weighted Supermatrix. To make the example more realistic, the other priority vectors were randomly perturbed to represent user error that will be introduced when making the other pairwise comparisons. 
Crit 1.1 Crit 1.2 Crit 1.3 Crit 2.1 Crit 2.2 Crit 2.3 Alt 1 Alt 2 Alt 3 Alt 4

\begin{tabular}{|r|c|c|c|c|c|c|c|c|c|c|} 
Crit 1.1 & 0 & 0 & 0 & 0 & 0 & 0 & 0.101 & 0.209 & 0.190 & 0.000 \\
Crit 1.2 & 0 & 0 & 0 & 0 & 0 & 0 & 0.223 & 0.140 & 0.143 & 0.000 \\
\hline Crit 1.3 & 0 & 0 & 0 & 0 & 0 & 0 & 0.162 & 0.215 & 0.119 & 0.000 \\
Crit 2.1 & 0 & 0 & 0 & 0 & 0 & 0 & 0.230 & 0.126 & 0.000 & 0.000 \\
Crit 2.2 & 0 & 0 & 0 & 0 & 0 & 0 & 0.132 & 0.215 & 0.000 & 0.000 \\
Crit 2.3 & 0 & 0 & 0 & 0 & 0 & 0 & 0.154 & 0.096 & 0.000 & 0.000 \\
Alt 1 & 0.165 & 0.477 & 0.227 & 0.176 & 0.158 & 0.311 & 0 & 0 & 0 & 0 \\
Alt 2 & 0.374 & 0.352 & 0.382 & 0.059 & 0.322 & 0.318 & 0 & 0 & 0 & 0 \\
Alt 3 & 0.316 & 0.105 & 0.067 & 0.294 & 0.268 & 0.133 & 0 & 0 & 0 & 0 \\
Alt 4 & 0.146 & 0.067 & 0.324 & 0.471 & 0.252 & 0.238 & 0 & 0 & 0 & 0 \\
\hline
\end{tabular}

The results from the original data (Actual) which included the correct priority vectors are presented next to the final results obtained by raising the updated weighted Supermatrix to powers to calculate the limit matrix. The updated weighted Supermatrix was obtained after the incoherent priority vectors, both the "intentional" which were left blank to reduce the number of needed comparisons and unintentional, were identified and updated using the LCI and LE from the original weighted Supermatrix. The final results are relatively close to actual results which is promising given the fact that error was introduced into the other comparisons. The LCI and LE can be calculated not only to test the coherency of the weighted Supermatrix but also to reduce the number of needed comparisons in an ANP decision.

\section{Final}

\begin{tabular}{l|c|c|}
\multicolumn{1}{c}{} & \multicolumn{1}{c}{ Actual } & \multicolumn{1}{c}{ Results } \\
\cline { 2 - 3 } Alt 1 & 0.276 & 0.267 \\
\cline { 2 - 3 } Alt 2 & 0.339 & 0.327 \\
\cline { 2 - 3 } Alt 3 & 0.181 & 0.188 \\
\cline { 2 - 3 } Alt 4 & 0.205 & 0.218 \\
\cline { 2 - 3 } & &
\end{tabular}

\section{Limitations}

Coherency testing is a novel way to improve the quality of ANP decisions. Because it is relatively new there is a significant opportunity to build upon and extend the initial method.

- Improvements may come in the form of using different distance metrics and/or aggregation techniques to calculate the LE and/or LCI.

- Using LE to reduce the number of comparisons in ANP decisions is just beginning to be explored; and there are many considerations that must be addressed to ensure that meaningful results are obtained.

- There will be a tradeoff between how many comparisons are made by the decision maker and how many are suggested by an algorithm and the potential impacts on the quality of the data. 
- It is not clear yet which parts of the Supermatrix are more important than others for the LCI and LE and for comparison reduction.

- Are there other connections among the elements in the Supermatrix that can be used to find and test even deeper relationships among the data?

- What new insights can Coherency provide regarding the inner and outer dependence in the Supermatrix?

\section{Conclusions}

Calculating the LE and LCI for the weighted Supermatrix in the example provided useful information to correct the priority vectors in the weighted Supermatrix before calculating the limit matrix. Coherency is a necessary condition to increase decision makers' confidence in their models. The ability to also reduce the number of comparisons needed to make an ANP decision has significant practical implications that can increase the use and application of the ANP.

\section{Key References}

Brunelli, M., Canal, L., \& Fedrizzi, M. (2013). Inconsistency indices for pairwise comparison matrices: a numerical study. Annals of Operations Research, 211(1), 493-509.

Cooper, O., \& Yavuz, I. (2016). Linking validation: A search for coherency within the Supermatrix. European journal of operational research, 252(1), 232-245.

Saaty, T. L. (1977). A scaling method for priorities in hierarchical structures. Journal of mathematical psychology, 15(3), 234-281.

Schoner, B., Wedley, W. C., \& Choo, E. U. (1993). A unified approach to AHP with linking pins. European Journal of Operational Research, 64(3), 384-392.

Yavuz, I., \& Cooper, O. (2017). A dynamic clustering method to improve the coherency of an ANP Supermatrix. Annals of Operations Research, 254(1-2), 507-531. 